Cuadernos de Filología Italiana

ISSN: 1133-9527

http://dx.doi.org/10.5209/CFIT.56701

\title{
Nelo Risi visto da vicino
}

\author{
Giovanna Ioli
}

Ricevuto 12 luglio 2017 / Accettato: 19 dicembre 2017

\begin{abstract}
Riassunto. Nella prima sezione di questo testo si analizzano le "esperienze" che stanno all'origine del lavoro di Nelo Risi. La trama dei punti di vista che s'irradiano nelle opere successive è il tema delle altre sezioni, dove sono messe in evidenza le occasioni pubbliche e private che forgiano la particolare forma espressiva e la magnetica memorabilità dello stile di Risi. La novità di questo percorso, in parte già noto, sta nel presentare l'autore seguendo il filo di un'inedita documentazione epistolare e iconografica, una "corrispondenza" d'intrecci e motivi che talvolta diventano ravvicinati, perché sarà la parola di Risi a irrompere dai fogli di un carteggio, mostrando come in uno specchio il suo profilo di uomo e di poeta.
\end{abstract}

Parole chiave: Nelo Risi; epistolario; Lucini; iconografia; Montale.

\section{[en] Nelo Risi analyzed closely}

\begin{abstract}
The first section of this text analyzes the "experiences" that give rise to Nelo Risi's work. The remaining sections first examine the weaving of perspectives in his subsequent works, and secondly underscore the public and private occasions which shape the particular expressiveness and magnetic memorability of Risi's style. This study is original in that it introduces the author by following the thread of an unpublished epistolary and iconographical documentation, a "correspondence" of intertwined strands and motifs that sometimes converge to each other. Risi's word will definitively break out from the papers of such correspondence by showing his profile of both man and poet as if they were reflections from a mirror.
\end{abstract}

Key words: Nelo Risi; epistolary; Lucini; iconography; Montale.

Sommario: 1. Profilo generale 2. Il Medico di Lucini. Lettere di Arnaldo Risi 3. Risi e Montale 4. Altro da dire. 5. «Rispondenze» epistolari 6. Riferimenti bibliografici.

Come citare: Ioli, Giovanna (2018) «Nelo Risi visto da vicino», Cuadernos de Filología Italiana, 25, pp. 247-265.

\section{Profilo generale}

Nelo Risi è un autore tra i più alti e coerenti della generazione postmontaliana, quella che usciva dalla guerra e dimostrava "altro da dire" da un punto di vista stilistico, affrancandosi dai codici dei vari ismi che hanno attraversato il secondo Novecento. Le formule per lui coniate dalla critica nel corso degli anni (stilista dell'usuale, poeta civile, linea lombarda o "laghismo" d'impronta sereniana) non riuscirono mai a rappresentarlo per intero. Risi, infatti, è sempre rimasto a latere dalle etichette d'an- 
tologia, rivendicando un personalissimo dettato poetico, nel quale agirono certamente molte influenze, domestiche e d'oltre confine: gli studi di medicina, le esperienze della guerra sul fronte russo, la scuola di Friburgo che accolse i rifugiati che si sottraevano alle persecuzioni belliche e la familiarità con la macchina da presa. In questo campo operò per molto tempo privilegiando "il reale" con documentari e inchieste televisive, per poi lavorare sull'immaginario dei film di finzione, acquistando infine la consapevolezza che anche il cinema «non è poi così lontano dalla poesia, un'immagine e poi un'altra e un'altra ancora... un verso e un altro ancora. A volte le regole del cinema facilitano la scrittura, e viceversa» (Risi 1988: 34).

Dopo il «tonfo», come Montale in Satura definì la liberazione e il dopoguerra, furono proprio le esperienze cinematografiche a spingere Risi verso un'attualità diversa, nel senso non solo del vero, ma di una cronaca che nei versi si trasfigura in un eterno presente, espresso in una forma aristocratica di "civilissima" trasparenza".

Dopo Le opere e i giorni del 1941, il primo passo di un viaggio letterario scandito da un'alternanza di silenzi rilkiani, Risi impresse nell'Esperienza gli effetti di una guerra che si può riassumere con Tutta polvere, il titolo del componimento che aprirà la raccolta successiva, Polso teso, dove i poeti piangono «e scrollano / fumo e polvere di dosso. / Non guariranno mai». È tuttavia curioso osservare che nel foglio della nota editoriale che anticipa il contenuto di «questo ideale carnet di viaggio», c'è anche una criptica indicazione biografica nella quale si legge: «L'autore (nato a Milano il 1920) è al suo primo libro, che però non è il solito primo libro di versi» (Risi 1948)².

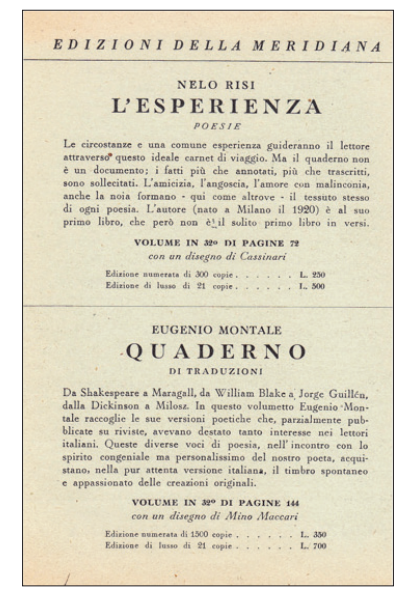

Si tratta di una notizia scarna ma anche monca, perché non cita il libro precedente; e una sorte simile avranno anche le trentacinque poesie della seconda raccolta, mai ristampata, che rimarranno fuori dai margini filologici degli studi critici. Quindici componimenti dell'Esperienza, infatti, che evidentemente Risi considerò come un lavoro in corso, confluiranno nella prima sezione di quella che lui considerò la vera opera prima: Polso teso del 1956, recensito con intelligenza da Raboni, mentre per le altre il cammino restava ancorato a quella proda:

Civilissimo 1 e Civilissimo 2, titolo di due sezioni della raccolta Pensieri elementari (Risi 1961). Civilissimo uscì anche in edizione numerata (Risi 1958).

2 Lo stesso foglio annuncia anche la pubblicazione del Quaderno di traduzione di Montale. 
Vent'anni ormai mi dividono dalla nascita parigina del libro, addirittura trenta per quella sezione che porta il titolo, dopo imposto, le "Vacche magre" che contiene poesie di una precedente raccolta; un intervallo tale da mettere alla prova questi versi. Rileggendoli ho cercato di comportarmi come il postero di me stesso; l'occhio attento al meglio, ho soppresso alcuni testi, ne ho scritti altri pochi, ho corretto dei versi, ho mutato una parola o un ritmo interno, ho alleggerito qua e là punteggiatura: insomma un lavoro di varianti doveroso a distanza di anni, anche se non ho voluto lasciarmi prendere troppo dal gusto del restauro (Risi 1956: 11).

Come "postero di se stesso" Risi agì per tutta la vita, senza dilungarsi in avvertenze e segnalazioni filologiche per il lettore, un esercizio che diventerà un labirintico rovello per gli studiosi armati di strumenti di diversa natura da quella che lui esprime in tutta la sua opera. Il Nelo Risi uomo, infatti, si specchia nei suoi testi, considerati un organismo vivente che cresce, cambia, pur rimanendo sempre se stesso. In altri termini, per lui anche la filologia diventa fisiologia che risponde solo a un'immanenza biologica, come se fosse un corpo con un suo codice genetico. Non è un caso che Risi abbia siglato quella che lui volle considerare la sua "prima" raccolta di versi con un titolo non metaforico: Polso teso, appunto, accelerato dal battito cardiaco.

Un analogo distacco si riscontra per una fiumana di scritti che non si curava di annoverare nelle scarne notizie bibliografiche che lo riguardavano o che restavano per decenni nel cassetto. Penso in particolare all'attività saggistica che dagli anni Quaranta appariva su riviste come Domus o il Politecnico di Vittorini, ma anche come introduzione ai numerosi poeti da lui tradotti o commentati.

Nel caso dell'epistolario del padre con Lucini, il distacco dalle vicende editoriali è esemplare. Il 24 aprile del 1998 scriveva:

Passo giorni di accidia e non produttivi. Sto chiudendo con Vanni [Scheiwiller] un "Lucini" fermo (incredibile) in una cassa da trent'anni, incompleto e che mi tocca ricostruire. Pare esca a Giugno, e l'avrai. Altro non faccio. Sì, ho curato un "Laforgue" per Garzanti in ed. popolare, e questo pure avrai. Vivo, come diceva Eusebio al $5 \%$. E scrivo con un occhio solo: si vede, vero?

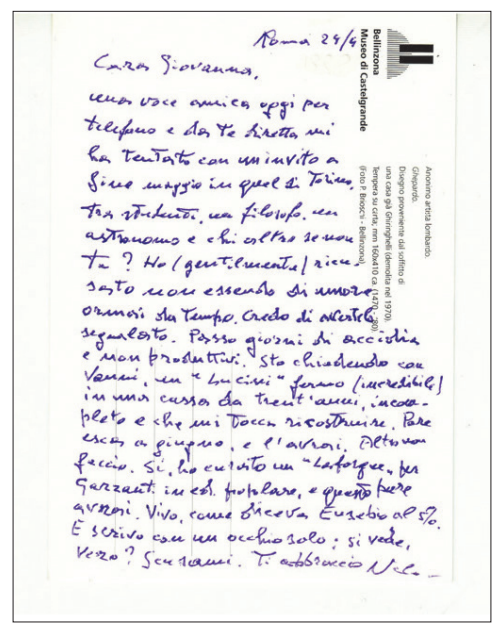


Con quell'occhio di straordinaria acutezza Nelo ricostruì il carteggio del padre, il dottor Arnaldo Risi (amico di Marinetti e poi esecutore testamentario di Gian Pietro Lucini, il poeta scapigliato-futurista, propugnatore del "verso libero" in Italia e poeta di forte vena civile), che «apparteneva a quella schiera di medici umanisti, di cui si va perdendo traccia» ${ }^{3}$. Tra le poche cose che Nelo conservava in una cartellina del suo studio c'era anche un foglio con dedica autografa, staccato dalla raccolta di elzeviri di Marinetti, Les Dieux s'en vont / D'Annunzio reste, "A mon très cher ami Arnaldo Risi hommage d'un vive admiration litteraire. F. T Marinetti $»^{4}$.

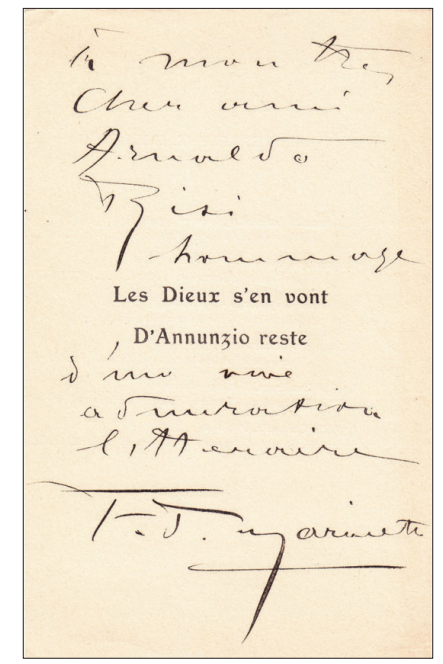

\section{Il Medico di Lucini. Lettere di Arnaldo Risi}

Le ripetute citazioni da opere diverse, saggistiche o epistolari, annotate da Nelo Risi (1999) nella lunga «Prefazione» a quel carteggio, diventano rivelatrici di un carattere che si forgia soprattutto su parentele di pensiero e ambizioni considerate eccentriche per i «pedanti ed i cattedratici da una parte, coi futuristi ed i deliranti dall'altra, per via di quel buon senso che non mi è lecito gettar via...». Gian Piero Lucini respirava un'epoca nella quale «gli uomini sono tutti malati», come diceva Ippocrate e di questa massima, scrive Nelo, «Lucini s'è fatto una regola di vita. [...] Lo stile, per Gourmont, "è un prodotto fisiologico" in diretta dipendenza dalle funzioni vitali», e quel poeta aveva formulato il modo per esprimerla. La visione aristocratica dell'arte di Lucini, allergico a tutte le forme e orgoglioso della sua misantropia «assetata di bellezza», che considerava una grande ricchezza la «libertà che non posso affittare», rispecchiava in qualche modo anche quella di Nelo Risis ${ }^{5}$.

3 Il carteggio tra Lucini e il suo medico curante uscì nella collana di Scheiwiller nel 1999, un libro che ha «una storia editoriale incredibile, che risale al '70, quasi trent'anni fa, quando Nelo Risi mi affidò il carteggio». Così scrive Vanni Scheiwiller nell'«Avvertenza». Si tratta di centodue lettere e cartoline che Lucini inviò al suo medico curante durante i quindici anni della loro amicizia.

4 Les Dieux s'en vont D'Annunzio reste fu pubblicato a Parigi dalla Bibliothèque Internationale d'Editions E. Sansot et Cie nel 1908, con copertina e tavole illustrate (per lo più caricature di D'Annunzio) del pittore Valeri.

5 Cito diffusamente dalla «Prefazione» di Nelo Risi (1999: 7-35, 140). 
Nella «Prefazione», infatti, che registra la cronaca della temporanea collaborazione di Lucini con Marinetti, iniziata nel 1902, emerge con evidenza che Nelo avrebbe poi ravvisato in lui un'impronta civile che sta anche alla base del suo personalissimo stile. Certamente non gli era sfuggito il vigore del poeta nel rivendicare un primato: avere intuito la forza rivoluzionaria dell'abbattimento dei confini metrici e la conseguente riscoperta di un'arte unica, capace di assommare in sé suono, immagini e contenuti ${ }^{6}$. Per questo motivo Lucini aveva ceduto al richiamo di Marinetti, con la speranza di ottenere un giusto riconoscimento del suo ruolo di precursore.

Ci tengo che egli [Marinetti] sappia, e vada a dire agli altri - scrive in una lettera del 1905 -, che la paternità del verso libero italiano mi appartiene esclusivamente, e che sono ben quindici anni che io mi sono fatto su quel règime di prosodia un mio verso personale, sicuro, e completo alla manifestazione del mio pensiero poetico: che fin'ora nessuno su questa via mi ha superato, né meno d'Annunzio col suo falso verso delle Laudi; come nessuno ancora ne ha saputo la secreta composizione e quel rispondere come fa all'armonia, al colore, ed alla densità del concetto che lo riempie come una guaina di maglia veste un corpo di bella giovine ${ }^{7}$ (Risi 1999: 140).

Marinetti aveva fiutato subito il vigore compresso nella poesia civile di Lucini, che andava "lancia in resta" contro borghesia, trono, altare, alla ricerca del rinnovamento sociale anche in sede letteraria, senza peraltro rinunciare alla tradizione per lui capeggiata dall'amato Foscolo. Dopo alcuni anni, però, ormai travolto dal seguito rivoluzionario del suo movimento, Marinetti non si rese conto che Lucini, come annotò Dossi nelle Note azzurre, «dalla Natura condannato al gabbo e alla rachitide, come dal Genio alla fama, aveva carattere subitaneo, iroso e, benché giusto, violento», al punto da sbattere la porta davanti alle opportunità che gli erano offerte ${ }^{8}$. La possibilità di una maggiore risonanza s'infranse così sullo scoglio della sua avversità per la letteratura di consumo e sul rifiuto di allontanarsi da una linea maestra che rifiutava i compromessi. Con la stessa stoica determinazione, allora, con la quale affrontò la mutilazione di una gamba che tenne sempre imbalsamata al suo fianco, non esitò a troncare anche la possibilità di uscire dall'ombra e il suo monumentale Verso libero resterà - disse Sanguineti (1998: 20) molti anni dopo - confinato come un trofeo «per bibliofili esigenti» e niente di più . Fu il primo transfugo del futurismo poetico di quella stagione. In una delle ultime lettere al suo medico, del 3 gennaio 1914 (morirà il 13 luglio, mentre corregge le bozze dell'Antimilitarismo), esprime l'amarezza e la delusione di quell'esperienza e sceglierà di essere

6 «Il Verso libero è la lunga parola poetica che esplica e chiude un concetto nella sua forma, nel suo calore, nella sua armonia, come nasce direttamente nella mente del poeta. È il mezzo per cui senza dispersione e senza aggiunte un pensiero è manifesto. Deve quindi essere pittura, scultura, musica, suggestione» (Lucini 1971: 117).

Lettera del 28 dicembre 1905. Cfr. anche Ioli (2011: 303-319).

8 Cfr. Dossi (1964, nota 5732). A Carlo Dossi, Lucini aveva dedicato il saggio L'ora topica di Carlo Dossi. Saggio di critica integrale (Lucini 1911 [1973]). Il carattere rivoluzionario del movimento futurista in fatto di arte e letteratura, coniugata alla loro posizione politica e reazionaria è stato ampiamente studiato e documentato in d'Orsi (2009).

9 Una stampa anastatica del Verso libero nell'edizione 1908 è stata pubblicata in occasione del centenario per le edizioni di Interlinea (Lucini 2008), a cura e con un saggio di Pier Luigi Ferro (2008: vii-xxxvii). 
pressoché ignorato alla totalità degli italiani senza speranza di diretta rimunerazione, in battaglia coi pedanti ed i cattedratici da una parte, coi futuristi e deliranti dall'altra, per via di quel buon senso che non mi è lecito gettar via, e senza di cui l'uomo è meno di un cane (Risi 1999: 187).

Dopo aver letto Il medico di Lucini, scrissi subito a Risi formulando l'ipotesi che nella sua «Prefazione» ci fosse anche una forma di rispecchiamento esistenziale che gli permetteva di comprendere pienamente lo stato d'animo di un poeta e le trame che avvolsero personaggi fuori canone come Lucini. La sua risposta non si fece attendere:

Cara Amica, anzitutto un saluto dall'amico a cui l'amicizia giova, altrimenti come avrei potuto ricevere l'intensa missiva che ha per tema, da un carteggio luciniano, le affinità paterne da cui conseguono alcune giudiziose considerazioni sul mondo letterario di cui facciamo parte pur non facendo nulla per esserne parte, fragorosamente emergente l'espace d'un matin? (30 maggio 1999) ${ }^{10}$.

Poi, com'era suo costume, cambiò subito discorso, archiviando un argomento che lui sapeva riassumere con quattro parole, seguite da un punto interrogativo, che traducevano i versi del suo amato Dante: «... Oh vana gloria de l'umane posse! / com' poco verde in su la cima dura» (Purg. XI 91-92).

Ho appena letto le tue pagine su Soldati e ho ritenuto un tuo giudizio su certe "descrizioni paesaggistiche" che sono "veri e propri talismani per continuare a vivere". Non è un complimento da poco, e si allaccia a quell' amore della parola che è il vero tema della tua lettera. Altro che "felice inutilità" altra tua espressione, questa corrispondenza a distanza riempie per qualche istante la vita.

Negli anni che si avvicinavano al fatidico 2000, la sensazione di vivere «al 5\% come Eusebio», diventò un ritornello ma Risi continuò a viaggiare a vele spiegate su un mare di carta, con una curiosità che sembrava insaziabile. Tutto quello che leggeva diventava spunto per una riflessione che lo riguardava "da vicino", spingendolo a rivedere i conti di un inventario personale che si traduceva in versi. Basta citare una lettera del 12 giugno 1999, dove traccia in poche righe un itinerario esistenziale diamantino, che non contempla ambizioni ma esperienze, perché era ancora il verso dantesco («Non è il mondan romore altro ch'un fiato», Purg. XI 100) il ritornello che da sempre scandiva il suo cammino.

Carissima, "Montale Solmi Zanzotto", il mio mondo, che è anche il tuo, che ti appartiene per lunga frequentazione e intelligenza di scrittura (come vado scoprendo, e dovevo sollecitarti) - e io che ignoravo l'esistenza di «Sigma». Ahimé che non ho mai fatto parte di nessuna consorteria! Fellini incontrandomi diceva: "ecco il mio poeta"; Caproni: "beato te che fai cinema"; il mio portiere dandomi la posta: "Dottore ricordi di farmi l'endovena"; a Parigi mi credevano pittore e i

10 Tutti frammenti epistolari che qui si pubblicano sono inediti e provengono dall'archivio privato di chi scrive che comprende lettere e cartoline di Nelo Risi dal 1991 al 2014. 
quadri erano di mia moglie - per anni ho girato il mondo da documentarista. Ho vissuto al 95\%. Oggi ho scritto i primi quattro versi del mio nuovo libro:

\section{Cercava Dio incerto se trovarlo in una pietra del deserto $\mathrm{o}$ in qualche ripostiglio della sua mente dispersa.}

E non so - io agnostico - se li userò. Grazie di avermi incluso nel "sogno" zanzottiano $^{11}$.

Il "sogno" di Zanzotto citato nella lettera era legato alla memorabilità dei suoi ritmi, che passavano come per un principio dei vasi comunicanti in altri poeti. In quel "sogno" avevo ravvisato, per esempio, la sincronicità junghiana di Giù per li rami di Minime massime (1962), che somigliava al "sogno" di Zanzotto in Microfilm (uscito in Pasque nel 1973, ma il componimento reca la data del 26 ottobre 1963). Potrebbe essere un altro esempio il verso «la meglio gioventù» della poesia di Risi Datata da Colonia del luglio 1948, dove allude ai giovani mutilati dalla guerra seduti sulle panche lungo il Reno, che scivolerà in un titolo di Pasolini ${ }^{12}$. E non è sfuggito a critici come Mengaldo che anche la svolta di Montale, da Satura in poi, sia stata in parte ispirata dalla poesia di Nelo Risi. Nel 2003 John Butcher gli chiese esplicitamente quale fosse il suo parere e lui rispose secondo il suo stile:

Io conoscevo bene Montale. Quand'era senatore a vita, per un periodo di tre o quattro anni, veniva a mangiare da me e mia moglie Edith Bruck. Il rispetto che ho avuto per Montale è stato immenso: non c'è dubbio che egli è stato il poeta che ha maggiormente condizionato il secolo italiano della poesia. Allora credevo che l'idea che noi poeti più giovani avessimo potuto suggerirgli qualcosa fosse piuttosto supponente. Di conseguenza in questo mio colloquio non con il "morto" ma con il "vivente" Montale, non ho mai voluto intavolare la questione della sua svolta poetica. Ma so che leggeva i nostri versi: so che ne ha tenuto conto. Ritengo che qualcosa abbia preso dalla mia poesia (Butcher 2003: 45-46).

\section{Risi e Montale}

Il nome di Montale ricorreva spesso nei nostri colloqui e, grazie a quel nome, lo incontrai per la prima volta nel 1991, quando mi fu affidata la curatela di un'edizione celebrativa per ricordare il decennale della morte. Si trattava di raccogliere una corona di poesie per testimoniare che «Montale ha camminato con noi, fra noi e con gli anni era diventato una guida o stava alla testa del gruppo di poeti e dopo la morte lo è rimasto, almeno nel profondo dei nostri spiriti e nel vivo della memoria, in una memoria trasformata in opera. [...] Non sono poesie d'occasione, sono documenti di vite intere, spese nella ricerca di voci e di modi sepolti, quasi si volesse

\footnotetext{
11 Il «nuovo libro» è Altro da dire, «composto in un arco di tempo che va dal settembre del 99 al marzo del Duemila». I versi citati nelle lettere sono inediti. Cfr. Risi (2000: 91). Per il «sogno» zanzottiano, cfr. Ioli (1995: 191-205).

12 Datata Colonia è nella sezione Viaggi e fatti personali (Risi 1956).
} 
dire: Se parliamo in questo modo è perché al fondo delle nostre speculazioni c'è stata anche, c'è stata soprattutto, c'era l'immagine positiva o negativa di Montale» (Bo 1991: ix-x). Per quell'edizione fuori commercio (18 poeti per Montale 19811991) Risi mandò un testo dal titolo Il poeta da vecchio, datato 14-15 giugno 1991, che rivedrà la luce nella sua ultima raccolta, Né il giorno né l'ora del 2008, senza alcuna indicazione temporale, con numerose varianti anche ritmiche e con un altro titolo, Sere della vita, che nell'originale figurava come capoverso. Questo dettaglio chiama ancora in causa il distacco di Risi da un ordine filologico o storiografico, come se tutta la sua opera appartenesse a un unico tempo nel quale riaffiorano a tratti momenti già registrati sulla carta, che in una nuova raccolta rappresentano un'evoluzione. E «il frutto della mia educazione» - diceva Risi nell'intervista a John Butcher - «sei anni di medicina sono formativi per un giovane. Se fossero stati quattro anni di filologia, io non avrei avuto la mia peculiare libertà di espressione» (Butcher 2003: 45). Era una sorta di «fedeltà al proprio io delle origini - osservava Mengaldo (1972: 857) - e il ricupero della propria storia più antica creando organismi poetici a vari strati e fondi».

Il 1991 era l'anno della pubblicazione di Mutazioni, «un libro di molte voci. [...] una minisumma umorale e risentita, tanti fatti che non hanno certezze» (Risi 1991: 91), sparsi in cinque sezioni, scortata la prima da una doppia epigrafe che segnala $i$ parametri geografici della sua formazione e dell'evoluzione del mondo: «La géographie de l'être a changé» da Michaux; e «...pur da mia natura / trasmutabile son per tutte guise!» da Dante, il poeta che fu sul suo scrittoio fino all'ultimo respiro. Come in una sequenza cinematografica, altri nomi propri scandiscono la sezione Una al giorno, che allude ai Fasti ovidiani, scritti in metro elegiaco seguendo il calendario romano. Anche il tono assume palesemente quello dei Tristia di Ovidio esiliato a Tomi: «Perdiderint cum me duo crimina, carmen et error» (Due crimini mi hanno perduto, un carme e un errore), annotava il poeta latino, cui fa eco anche la malinconia di Risi. La mutata fortuna di Ovidio diventa così titolo della seconda sezione della raccolta, anticipata da un'altra epigrafe, da Puškin: «la tua voce sconsolata ai luoghi ha dato lustro / dove ancora suona tenero il tuo canto». Da questo punto in poi, i testi sono impaginati al fondo delle pagine, come se nascessero da un vuoto, un venir meno della scrittura:

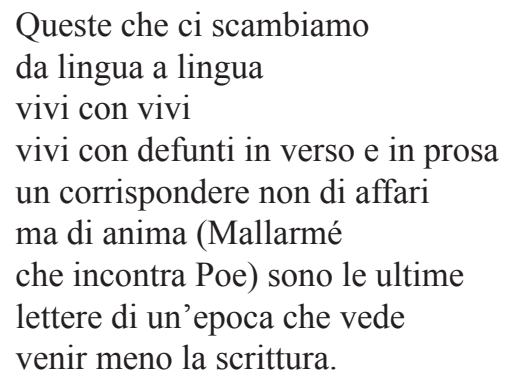

Per quella raccolta - che certificava una statura ormai consolidata armata di sarcasmo - l'anno successivo gli fu assegnato il Premio Librex-Guggenheim Eugenio Montale che rendeva omaggio alla coerenza e al rigore di un esercizio inventivo e che, già nei testi di Le opere i giorni (1941) e dell'Esperienza (1948), aveva assunto tratti di memorabile autonomia espressiva. 
La copia di Mutazioni che conservo, sigla con una dedica un'amicizia destinata a durare anche oltre la soglia che apparentemente oggi ci separa: «a Giovanna / che mi ha preso per mano / per condurmi al Premio». Era il 12 ottobre 1992 ed eravamo a Milano, la città della sua infanzia che cominciai a conoscere dalle storie che mi raccontò in quei giorni trascorsi insieme. Parlammo molto di Montale, ovviamente, del nostro modo di leggere $i$ testi di un poeta che era entrato a far parte della sua biografia e dei miei studi. Furono queste le premesse della lunga corrispondenza epistolare che si affaccia per accenni in queste pagine, non certo per esibire un "io" che non fa parte delle mie corde, ma perché Nelo possa riprendere il suo fiato, animando con voce confidenziale anche la marmorea fissità della scrittura.

Risi parlava di Montale come fosse una «parallela, ombra concorde» (le Personae separate della Bufera) e assumeva un analogo timbro anche nelle interviste, quando spiegava le direttrici della sua bigama formazione: per un verso la lezione di Rossellini, da lui considerato il più grande regista del cinema italiano, che gli insegnò a far rivivere la realtà lavorando con la finzione e, per l'altro verso, la lezione di Eusebio. ${ }^{13}$ Non è un caso raro per i poeti della sua generazione, mentre è eccezionale il contrario e, come accennato in precedenza, lo aveva già rilevato l'orecchio attento di Mengaldo nel 1971:

Lo stilismo dell'usuale di Risi, risposta decisa a una crisi storica di linguaggio e ideologia poetica, ha largamente alimentato le esperienze di molta poesia del dopoguerra che potremmo chiamare di "terza forza" (a sua volta traendone alimento) dallo stesso Fortini a Giudici a molti più giovani come Raboni, Bandini, Majorino ecc. (e perché no anche un "vecchio" come l'ultimo Montale?) (Mengaldo 1972: 857).

La poesia di Nelo Risi è stata, infatti, una sorta di magnete e certamente anche Montale ha sperimentato questa forma di attrazione. Tra le sue rare recensioni ai poeti contemporanei c'è anche Risi, due volte (come per gli Strumenti umani di Sereni, e La beltà di Zanzotto). La circostanza non si spiega soltanto con un reticolo di riferimenti biografici, ma obbedisce $\mathrm{a}$ un'intenzionalità più profonda. Soprattutto la seconda recensione del ' 66 e passibile già in funzione del quarto e quinto libro di Montale, le opere che segnano la differenza e discontinuità dalla Bufera. L'articolo, uscito sul Corriere della Sera del 6 febbraio 1966 aveva come titolo «L'enorme anonimato in cui tutti viviamo» ed esordiva facendo chiarezza sui libri di poesia «con titoli ambigui o decisamente antipoetici», portando come esempio il libro di versi di Bino Binazzi che s'intitolava La via della ricchezza, schedato in una grande biblioteca nazionale sotto la rubrica dell'economia.

I titoli scelti da Nelo Risi per i tre volumi da lui pubblicati nello «Specchio» mondadoriano: Polso teso, Pensieri elementari, Dentro la sostanza (l'ultimo) hanno però poca probabilità di essere registrati sotto le voci della medicina o della fisica. La proliferazione dei titoli antipoetici deve ormai aver suggerito la necessaria pru-

13 L'opzione onomastica di Eusebio per designare Montale è un attributo usato da Bobi Bazlen e richiama la natura di uno dei due personaggi di Schumann: battagliera ed eroica l'indole di Florestano; malinconica e fragile quella del più timido Eusebio. Con questi due nomi Schumann, a seconda del suo stato d'animo, usava firmare la sua musica e i suoi scritti sulla Neue Zeitschrift für Musik. Oltre che nelle Davidsbündlertänze, dove i due sono gli indiscussi protagonisti, Florestano ed Eusebio appaiono anche nel celebre Carnaval Op.9. 
denza anche agli schedatori. Inoltre i tre titoli riflettono a meraviglia l'arte poetica di Risi, i caratteri di una poesia che aspira alla tensione, alla semplificazione del mezzo espressivo e alla nuda sostanza di un sentimento che non è mai distaccato dalla riflessione. Si potrebbe credere a prima vista che Risi sia uno di quei poeti che partono dallo zero, facendo tabula rasa della loro cultura; [...] Nelo Risi non rifiuta affatto la sua natura e la sua formazione di uomo colto (Montale 1966: 11).

Bastano pochi passaggi delle pagine successive per capire che Montale aveva colto con precisione i nuclei fondanti dello stile di Risi, che «fa i conti con un tempo arido, con una stagione in cui tutto sembra detto; fa i conti con l'enorme anonimato in cui tutti viviamo, con la condizione storica dell'uomo-formica che sembra la tragica novità di oggi e di domani e forse è antica quanto l'uomo». Senza rinunciare alla sua natura di uomo colto - dice sempre Montale - Risi incide il suo bisturi "dentro la sostanza" delle cose e, senza essere «engagé nell'accezione ordinaria del termine, anche la sua indignazione» assume precisi «intenti politici». Chi ha dimestichezza con l'opera di Montale può riconoscere facilmente i riverberi di queste considerazioni che si accendono in Satura e alludo soprattutto al «formichiere» di Realismo non magico (datato 1969), ai «formiconi degli approdi» di Botta e risposta $\mathrm{I}$, al formicaio umano (datato 1968) di Diafana come un velo la foglia secca («e ora tutto è cambiato, un formicaio / vale l'altro»). E resta memorabile la risposta alle accuse di non essere engagé nella Lettera a Malvolio («non si trattò mai d'una fuga / ma solo di un rispettabile / prendere le distanze»). Montale sapeva bene che «Scrivere è un atto politico», come diceva Risi in Dentro la sostanza (1965), un libro nel quale raggiunge il diapason dell'impegno che da civico diventa l'amara analisi di un sistema da colonna infame che muta nella forma ma non nella sostanza. Così, nel lungo elenco delle Tavole del vecchio stato non rinnovato, oggi come ieri «Ogni uomo che sa / è una macchia che s'allarga (favorire l'inerzia) // Angelo che s'alza / in volo, va / abbattuto subito (sorvegliare le frontiere)». L'uomo, insomma, «Durante una vertigine di epoche / è ancora Adamo sempre più Caino (stupido e smarrito in controversie / in qualche oscuro argomento familiare, / bendisposto allo sterminio» (Arte poetica).

Nella prima recensione, uscita sul Corriere della Sera del 26 giugno1957, invece, nel recensire Polso teso Montale aveva tirato subito in ballo il Risi traduttore delle Poesie di Pierre Jean Jouve, che erano state pubblicate nello stesso anno con una presentazione di Ungaretti dall'editore Carucci di Roma. L'autore francese era nelle corde di Montale già ai tempi del Quaderno genovese, dove lo annovera tra chi ha avuto «il merito di innalzare l'arte, ponendola al di sopra delle anime mediocri» (Montale 1957). Non è ininfluente ricordare inoltre che Jouve nel 1964 tradurrà alcune poesie di Montale, poi pubblicate da Scheiwiller, il che giustifica la sintonia che s'instaura tra poeti di generazioni così diverse.

Al fiuto di Eusebio non sfuggì che quella di Risi è la poesia del detto e del pensato, del documento e dell'invisibile, del silenzio rilkiano, in un procedimento che non riguarda solo i contenuti, ma che si rispecchia fedelmente nelle "risonanze" del linguaggio con un «ritmo di vita parlata» (Risi 1970: 39) che non ha mai smesso di essere quello del colloquio, del borbottio sommesso di chi guarda il mondo dall'interno del suo guscio mobile, impotente ma deciso a resistere, a combattere con armi a lama sottile.

Di «resistenza della poesia e dell'esistenza» Nelo parlava nel giugno del 1946 sul «Politecnico» di Vittorini e questo suo "impegno" già in Polso teso $(1956,1973)$ 
aveva acquistato un'identità certa, senza ripensamenti. La scelta del linguaggio per esprimerlo senza fanfare precede la fiumana diaristica di Eusebio, così simile ai Segni dei tempi di Risi ${ }^{14}$, appunto, dove scriveva che «La storia è fatta di strati $\backslash$ chi la studia procede \secondo l'ordine dei depositi», e dove - tra il 1967 e il 1969 - si stendeva «un elogio uniforme della ragione [...] che attraverso ponderati sarcasmi e ricerche stilistiche tende alla comunicazione con l'uomo» (Risi 1991: 93).

Il verso che sia anche prosa rientrava anche nei giovanili sogni di Montale, ma il semenzaio necessario per intraprendere in via definitiva questa strada non lo trovò solo in Gozzano, ma in Risi, che non si lasciò mai corrompere da lirismi che non fossero quelli segreti di una musica, di un suono, che sapeva trovare solo nella lingua viva, composta in sillogismi irrefutabili, apodittici: «la poesia è verità $\backslash$ intuita con ritmo» (Post scriptum, Risi 1965)

Impermeabile ai manierismi sperimentali, alla psicologia di maniera, alle neoavanguardie, Risi ha sempre operato come un chirurgo, con un occhio clinico puntato sul reale, esaminato con meticolosa attenzione, organo dopo organo, come in un esame autoptico che sarebbe piaciuto a Gottfried Benn.

Fisicità che condurrà verso un'arte che si prefigge una biologizzazione dell'esistente, un vedere la scrittura con l'occhio del medico o con l'occhio del biologo, come in Benn, meglio ancora come in Musil che conduce alla fine dell'uomo che non parla più. Monologismo lirico che non attende risposta. C'è una mentalità tecnica da medico in quel "Polso teso" che ho sempre sentito iperemico, un di più di pressione sanguigna $[\ldots]$ nel mio scrivere in versi devono ancora essere rintracciabili dei potenziali chimici espressi nei cromosomi di mio padre: questa eredità biologica naturalmente non presumo che abbia una dominante, piuttosto un carattere recessivo intermedio o parziale come accade con l'età nel colore dei capelli, non come legge dell'eredità, piuttosto come fenomeno fisiologico. "Medicina” era per me un supporto prezioso di conoscenze, pur nella rinuncia della professione, che doveva aiutarmi a capire e interpretare le realtà in aree geografiche altrimenti estranee alla vita interiore (Risi 2009: 137-138).

Quella di Risi non rappresentava tuttavia la poesia prosastica tout court, perché «qui non si fa romanzo» (Risi diceva in Ruggine) ma poesia, con una prosa che ha l'anima dei versi. Per lui «la poesia è l'arte che vede. Una vita quasi ascetica (in un senso tutto intellettuale di solitudine) non disgiunta da una naturale inclinazione aristocratica», come scrisse nell' «Introduzione» alla nuova edizione delle Poesie di Jouve (2001) che ha come fil rouge la Commedia dantesca, «il cammino di Dante rovesciato» verso Il paradiso perduto, «dove l'eterno è una sete di assoluto che non ha bisogno di identificarsi necessariamente con Dio; può essere anche sentito come idea di un ordine che aspira continuamente ad attuarsi, e la vita come un continuo esercitarsi a morire» (Jouve 2001: vii) ${ }^{15}$.

Le traduzioni di Risi non sono ininfluenti per tracciare il suo profilo, perché ridefiniva e rinominava le parole altrui al punto da trasformarle in una realtà poetica sua.

14 Titolo interno che segnala i contenuti della raccolta Di certe cose.

15 Il paradiso perduto è un poema di Jouve uscito nel 1929 che, per volere dell'autore, è rimasto escluso dall'antologia tradotta da Nelo Risi. Sarà pubblicato, con un disegno di R. Birolli, Birolli, nelle Edizioni della Lanterna (Bologna, 1961). 
Jouve, Laforgue, Kavafis, soprattutto, sono altrettante personae separate, come le montaliane «aste di un sol quadrante» già citate. Varianti "fisiologiche" si ritrovano anche nelle traduzioni, che per Nelo Risi sono sempre nutrimento e partenogenesi, riproduzione virginale e "corrispondenza" di pensiero: un'opera-corpo originale da aggiungere alla bibliografia della sua poetica, che poteva mutare rinominando le cose e ridefinire il ritmo da un'edizione all'altra.

Non è un caso che, come tacito "lascito testamentario" a brandelli epistolari, il 26 marzo 2005 mi spedì tre fogli strappati dalla prima edizione della sua traduzione di Sueur de Sang (1933-1935), Sudore di Sangue, che contenevano otto liriche. Sul frontespizio Nelo Risi scriveva:

Per la nostra cronistoria:? / queste sono in assoluto le prime poesie da me tradotte all'età di 19 anni. Come dire, sono rimasto colpito da questa scrittura, di P.J Jouve. Avevo appena pubblicato la plaquette "Le opere e i giorni” grazie all'interessamento del "vecchio" Scheiwiller. / n.b./ "Poesie" di P. J Jouve p. 340 con testo a fronte è uscito presso l'Ed. Lerici nel 1963, a cura di N. R.

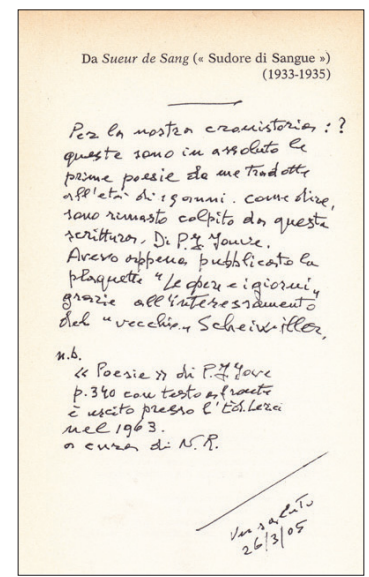

Anche la traduzione da Jouve rientrava nella nostra "comunicazione in parallelo", uno specchio nel quale riflettersi che mandava segnali luminosi che avrei dovuto interpretare. Nell'edizione del 2001, aggiunse un'introduzione nella quale si mostrano i motivi della sua lunga fedeltà a un autore del quale si riconoscevano i tratti e, com'era suo costume, anche una serie di varianti non sempre segnalate. Quando, anni dopo, accennai al suo distacco dagli orizzonti filologici, mi rispose: «Non sono un letterato (un oggetto scolastico). Semmai un elemento artistico pluriculturale autonomo, di varie esperienze anche a rischio, che mi hanno condotto in giro per il mondo - educato da medico, traduttore, scrittore in proprio, uomo di cinema legato all'immagine» (23 dicembre 2009). E come tale, quel genere di studi non rientrava nei suoi orizzonti, lasciando ai posteri un vasto materiale di ricerca per diligenti compilatori di un'edizione critica, che ci auguriamo di vedere un giorno rifiorire a stampa.

È un' ennesima prova della natura di Nelo Risi, forse la più libera del suo tempo dal rampante fenomeno pubblicitario, dall'arrivismo e dal carrierismo, dalla logica del profitto che non furono mai nelle sue corde. Edith Bruck, in un recente convegno organizzato dalla Fondazione Palmisano, disse che questa per lui era una regola di vita: 
la sua incorruttibilità, dignità, aristocrazia innata, la sua coerenza, la sua rettitudine, il suo dare senza chiedere, senza cedere a censure sui propri lavori televisivi pagandone il prezzo con periodi di disoccupazione. Lo amo per il suo pudore, umiltà dei grandi, ironia colta, discrezione e sensibilità. Per il totale disinteresse materiale e per la proprietà, i soldi li usa per i libri, i dischi; mi ripete che per un uomo basta coprirsi se ha freddo, mangiare se ha fame e avere un tetto sul capo. E così fu suo padre medico che curava i più diseredati, suo nonno ingegnere che ha lasciato ai cari figli un testamento etico morale, null'altro.

Lo amo per la sua libertà di dire, di agire, il suo impegno civile e la sua generosità ricca senza essere ricco (Bruck 2017: 30).

Così affrontò la vita riversata anche nelle pagine da lui scritte in sessant'anni, dove riaffiora una natura che rifiuta tutti gli effetti di superficie, ma anche l'ispirazione orfica o onirica, mostrando fin dagli esordi un approccio illuministico alla materia della sua poesia, che restava "dentro la sostanza" delle parole e delle cose. «A me piace nominare le cose - diceva in un'intervista - chiamarle col loro nome, e dai fatti trarre una morale o suggerirla. Sono un lombardo, la mia educazione è laica e illuminista; del Manzoni preferisco la Colonna infame ai Promessi sposi e mi trovo a rileggere con maggior passione il Parini che non il Foscolo. Sicuramente sono dotato di scarsa immaginazione e la mia natura è più riflessiva che creativa» (Risi 1981: 408-409).

\section{Altro da dire}

Non sempre quello che gli spedivo, seppure da lui sollecitato, suscitava il suo interesse, come nel caso - prevedibile - di un autore come Buzzati. Il 23 agosto 1999 scriveva:

Roma, di ferragosto: il postino, raro sopravvissuto, mi porta il tuo elaborato documentatissimo studio su "Buzzati" (che mai avrei sospettato parte del tuo mondo) ottimo giornalista cui ha nociuto il confronto con Kafka e che tu con generosità promuovi a maestro di linguaggio e a romanziere fantastico. Io lo trovo dotato (il suo militarismo di maniera) e tuttavia ti ho letto per intero e ancora una volta ne ho tratto il convincimento che la poesia (anche in quegli anni quaranta) stava un gradino più su della prosa. Dunque una lettura utilissima e che mi ha regalato una pagina di Rilke da te citato a p. 119-120 e che dovrebbe aiutarmi nell'impresa di finalmente mettere mano al mio libro per Mondadori. Grazie di questo suggerimento da sensitiva. Come sempre riusciamo a tendere un filo che non sia il telefono.

Pensai che fosse una formula consolatoria, ma esattamente due mesi dopo - il 23 ottobre 1999 - mi arrivò un'altra lettera che riprendeva il filo di quell'argomento:

[...] sono in ritardo col libro per Lo Specchio, che ha per titolo "Altro da dire" e che dovrei consegnare a gennaio del fatale Duemila. [...] È da un pezzo che desideravo "sentirti" - ero rimasto al Buzzati, col timore di averti delusa per il mio scarso apprezzamento verso il narratore. Alla lettura del tuo saggio devo comun- 
que la pagina dal Malte che metterò in apertura al mio quaderno di versi. Come sempre mi vieni, di riffa o di raffa, in aiuto.

Come per effetto della già citata sincronicità junghiana che lui conosceva bene, Altro da dire uscirà nell'ottobre «del fatale Duemila». L'epigrafe è di Rainer Maria Rilke e nella «Notizia» scrive:

dopo un silenzio di anni e un distacco totale dallo scrivere in versi il libro è stato interamente composto in un arco di tempo che va dal settembre del '99 al marzo del Duemila. Tutte le poesie sono inedite.

Altro da dire è nato su sollecitazione di Maurizio Cucchi, che ha sempre dimostrato un'affettuosa attenzione per la mia poesia.

Un grazie particolare va all'amica Giovanna Ioli, che mi ha incoraggiato a perseverare nell'impresa vincendo lo smarrimento iniziale, pescando nel disordine non programmato della mia esistenza.

Risi mi aveva mandato in anteprima il dattiloscritto di Altro da dire e avevo riversato le mie suggestioni in una lettera. È un libro, dicevo, che mette in moto gli occhi e il pensiero, una poesia per immagini con una perfetta sintesi di fotogrammi e misteriosi impulsi genetici. In quei componimenti agiva ancora il terzo occhio di Risi, quello ereditato dal padre, dal medico abituato a guardare oltre le superfici, in una istintiva anamnesi del male del mondo. Fanno apparentemente eccezione le due sezioni centrali («Altre dimore con figure» e «Figure»), avvolte dalla Storia, ma come sospese in una bolla di sapone, iridescente, dove sono i sintomi della tenerezza e della quiete a restare impressi nella cartella clinica di un'altra storia. Tanti poeti sono chiamati a raccolta nel filo narrativo che tiene insieme il libro e in modo dichiarato compare anche l'ultimo Montale, quello di «Satura per esempio dal linguaggio sottotono», lo stesso linguaggio che Risi aveva già esercitato vent'anni prima, quando Eusebio vedeva ancora fantasmi salutiferi nei riflessi del sole sulle acque delle Cinque Terre ${ }^{16}$.

Da quel momento la storia epistolare che già ci univa da anni subì uno scarto di registro, diventando «uno specchiare di umori attenzioni intuizioni critiche, soprattutto di rispondenze a senso unico che però ci comprendono». Lui spediva i suoi inediti, accompagnati da lettere che ne annunciavano altri («credo che sarai destinata a leggermi nel tempo pagina dopo pagina se questo mio esercizio poetico continuerà», «la corrispondenza si fa più fitta col pretesto - per parte mia - di inviarti una paginetta dei miei versi al giorno», «credo si stia delineando un disegno, ancora non so»): lettere alle quali rispondevo d'istinto, seguendo in corso d'opera la nascita di un libro (Ioli 2008). Dieci libri appena ristampati in uno solo riempiono una vita e potrebbero suggerire un esito definitivo, diceva, alludendo alla raccolta di tutte le poesie, curata da Maurizio Cucchi per gli Oscar Mondadori, Di certe cose (poesie 1953-2005), che mi spedì con una dedica nella quale annunciava: «ora non mi resta che creare il silenzio». Non si riferiva a quel volume, ma all'altro ancora inedito, musicato quasi per intero nel 2005 con armonie che confluiranno in Né il giorno né l'ora: una raccolta già pronta, che per faticose vicende editoriali vedrà la luce nel 2008 (d'Orsi 2017: 77-82).

16 Il verso citato è tratto da Che sia venuto il momento di rileggere Montale, in Risi (2000: 30). 


\section{Rispondenze epistolari}

«Uno specchio di umori attenzioni intuizioni critiche, soprattutto di rispondenze». Così Nelo Risi aveva riassunto la «comunicazione in parallelo» della nostra storia epistolare, durata «pochi anni ma così preziosi che meriterebbero un giorno di essere conosciuti» (12 novembre 2005). Sono queste le parole per accompagnare il mannello di lettere che gli avevo scritto negli ultimi anni e che lui mi restituì, perché temeva che dopo la sua partenza verso l'altrove andassero disperse, rivelando un latente desiderio di renderle pubbliche. In quella corrispondenza c'è anche la "cronistoria" della sua ultima raccolta. Dopo avere consegnato i testi di Ruggine all'editore diceva, infatti, di non avere davvero "altro da dire", ma poi ci fu un lampo che si accese, imprevisto, nella camera oscura di un museo. Da quel momento, come una montaliana cascata «di voci verso un esito», le lettere di Nelo cominciarono ad approdare copiose sulla mia scrivania.

Dallo spoglio a posteriori di tutto il suo carteggio, appare anche con evidenza quale fu la scintilla che avrebbe dato origine alla raccolta Né il giorno né l'ora, l'origine-vertigine che aprì una nuova vena nel cristallo della sua immaginazione: «una vertigine dall'invisibile / al visibile che affiora». Fu una sorta di "occasione" che, grazie a quelle lettere, permette di stabilire il giorno e l'ora in cui fu sfiorato da una sorta di folgorazione. Era il 17 ottobre 2003 e Nelo era Torino per una Tavola rotonda organizzata dall'Università per il bicentenario della nascita di Niccolò Tommaseo: Gli scrittori e il vocabolario. Fu lui a chiudere i lavori e la sua partenza per Roma era fissata per il giorno successivo. Come mi aveva chiesto, andai a prenderlo in albergo con largo anticipo e l'indecisione su come trascorrere le poche ore a disposizione durò un attimo. La sua attenzione, infatti, si concentrò subito sul manifesto che campeggiava nella hall dell'albergo: una mostra di capolavori del continente africano ospitati dalla Galleria d'arte moderna di Torino. Quindici minuti dopo ci trovammo immersi in una sorta di incantesimo affollato di pensieri, di emozioni, di sentimenti così sorprendenti da trasformare quella visita in un evento. Nel buio delle sale le millenarie sculture sembravano apparizioni, souffles di anime che rivendicavano la loro esistenza. Nelo si avvicinò a una figura umana con le braccia alzate dell'IX-XI secolo e cominciò a leggere in un sussurro la didascalia. Erano versi di Birago Diop, un poeta senegalese impegnato nella trascrizione della letteratura orale locale, che spiegava perché I morti non sono morti:
Ascolta più spesso le cose
Che gli Esseri.
La voce del Fuoco s'intende, ascolta la voce dell'Acqua.
Ascolta nel Vento
Il Cespuglio in singhiozzi:
È il respiro degli Antenati.

Nelo aveva filmato nei suoi documentari le situazioni e i conflitti nel Sahara già negli anni Quaranta, al seguito di due grandi reporter cinematografici, Richard Leacock e John Ferno. Aveva poi girato il documentario Eritrea si parte da zero, prodotto dalla RAI nel 1997, ma non aveva dimenticato la desolazione di una terra sfregiata da altre morti, come testimoniano i pochi lancinanti versi di Altro da dire 


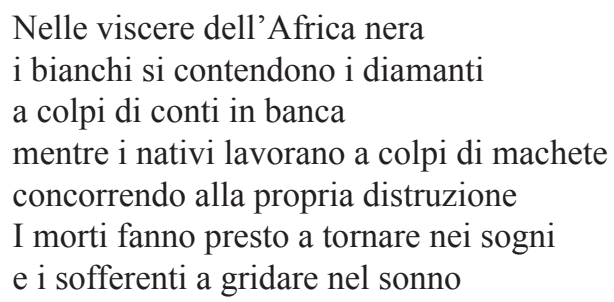

Appena tornato a Roma, mi scrisse una prima lettera di ringraziamento e poi una seconda per chiedermi di tornare alla Mostra dove acquistammo i cataloghi per chiedere alla commessa se erano in vendita anche libri di Birago Diop. Ovviamente non li trovai, ma continuai a cercarli e il 23 novembre $2003 \mathrm{mi}$ scrisse:

i racconti appena ricevuti di Diop mi riportano ai capolavori [...] che tanto c'incantarono in quel mattino d'addio e che sono tuttora una della maggiori emozioni di questi tempi così poco fiabeschi. Statuaria lignea e bronzea del Dogan, del Mali e di quanto quel territorio sub sahariano ha saputo creare in secoli non troppo lontani e offerti alla nostra incantata ammirazione. Nella penombra sacrale quasi non si parlava; come pure il viaggio fortunatamente eterno sul ritorno ha finito col riempire i miei occhi chiusi su quelle immagini. Mai treno fu più ricco.

Il primo febbraio 2004 quel viaggio nel buio era già impresso in un lungo testo intitolato Trasferta museale, seguito da una dedica ("a Giovanna"), che confluirà con diverse varianti in Né il giorno né l'ora ${ }^{17}$. Il 3 febbraio gli giunse la mia risposta, ammirata dalla stupefacente vitalità di un componimento nel quale già respirava il risveglio della sua poesia, che si opponeva al suo sempre annunciato silenzio. Il 4 febbraio scriveva: «verrebbe voglia di provare a scrivere giornalmente dei versi per ricevere missive di questo tenore». Il 6 febbraio rilanciavo l'idea: una poesia al giorno, un diario diverso da quello montaliano, con «altre vibrazioni, un contraltare di buio e di luce, con la tua capacità impeccabile di sfiorare letteratura e vita, di renderla visibile e sottrarla subito alla difesa, come fa la "sensitiva" di Montale o l'anemone di mare sui coralli». Il 10 febbraio già ricevevo un nuovo testo, Tholos, un altro «Itinerario fatale» della futura raccolta ${ }^{18}$.

Nei mesi successivi il botta e risposta cambiò direzione perché i torchi della Mondadori erano pronti per la pubblicazione di Ruggine. Il 25 marzo 2003 mi aveva spedito il dattiloscritto, accompagnato da una lettera nella quale diceva di avere ricevuto un contratto che stabiliva la data di pubblicazione del suo «quaderno rugginoso»: il primo trimestre dell' anno a venire, «come fossi immortale... Sento il tempo che si restringe come un pachidermico che ha scelto di appartarsi nel folto e rifiuta compagnia».

Era convinto che quella fosse davvero la sua ultima raccolta di versi, ma il 20 gennaio del 2004 mi spedì Passo passo..., come per dire che dopo la "trasferta" torinese la storia non finiva con Ruggine e che aveva intrapreso un nuovo cammino. La data della lettera è indicativa, perché, dopo avere affermato per l'ennesima volta di non avere più «niente da dire», aveva ripreso a scrivere versi «in cerca di un nonfinito» (Rendiconto), «nell'indistinto / nel flusso riflusso di un futuro», annunciando un nuovo dizionario «per comprendere la morte / come nuovo elemento di vita» (Nessuno).

\footnotetext{
La poesia uscì in anteprima tra gli Inediti (Risi 2005: 7-9).

«Itinerari fatali» è il titolo dell'ultima sezione di Né il giorno né l'ora.
} 
Seguirono altre lettere, a cascata, un esercizio che il 7 giugno 2005 Risi definiva in modo programmatico:

credo che sarai destinata a leggermi nel tempo pagina dopo pagina se questo mio esercizio poetico continuerà.

Non so esattamente perché scrivo e dove queste riflessioni mi porteranno. Forse sono arrivato al punto che ho bisogno di uscire da questo mondo che mi va sempre più stretto e che non sopporto più...

In allegato c'era la poesia Metamorfosi o la piacevolezza del mito, con diverse varianti nella versione a stampa. Dopo una settimana, ripeteva la stessa intenzione:

la corrispondenza si fa più fitta col pretesto - per parte mia - di inviarti una paginetta dei miei versi al giorno. Ieri la tua lettera mi ha camblé perché hai accolto con favore la "Metamorfosi" di cui non ero convinto della riuscita, anche perché sono in cerca di una svolta tematica anche nel linguaggio. Ieri Págo ero fisso al bello. Oggi ti sottopongo qualcosa che mi fa tornare al mio "Ruggine" e di cui ignoro l'esito, che non vorrei scontato. Com'è difficile trovare equilibrio nel nuovo.

Il nuovo testo aveva come titolo Come lottare con il futuro (Un futuro, nell'edizione a stampa), dove la novità stava nelle sillabe, compresse al punto da trasformarsi in ossidiana. Ne scaturiva un ritmo speciale, una misteriosa convergenza di suoni interni, una specie di musica del silenzio che spandeva un alone di malinconica adesione alle forme, alla sostanza delle sillabe. Gli scrissi che mi sembrava di vedere mani che carezzano la sofferenza, sguardi sulle cose, sul mondo, sulla vita che passa, lievi come un frullo d'ali (16 giugno 2005).

Quattro giorni dopo la sua risposta era già arrivata:

credo di avere detto recentemente in un'intervista che io sono un uomo con delle fortune. Una, l'ultima in ordine di tempo, è quella di averti conosciuto. Non i miei "minima moralia" contano ma i tuoi scritti a delucidazione di quanto espresso, da "Altro da dire" a "Ruggine" e ora in queste carte non ancora ordinate che ti spedisco. Hai una capacità davvero insolita di interpretare e penetrare quanto ti invio portando giudizi immediati che via via mi definiscono e fanno da guida al pensiero a venire.

Ecco perché ho inventato e proposto quell'una al giorno che non avrebbe senso per me se io non attendessi il chiarimento di ciò che via via vado scrivendo. La tua del 16 , sotto i miei occhi mentre scrivo, è particolarmente suggestiva quando delinei quella musica del silenzio che cerco di inseguire sillabando in modo sempre più conciso i miei respiri mentali. Che tu sia telepatica mi è noto da tempo; non al punto di suggerire una guida al sentire in fieri. Tu dai aria con il tuo sguardo in anticipo alle cose del mondo. Quanto al "frullo d'ali" in questi ultimi tempi si vola da Roma a Torino e ritorno.

Nel giro di un anno, lettera dopo lettera, la nuova raccolta era finita, ma dovette aspettare tre anni prima di vederla a stampa, accompagnata da un'introduzione che, in virtù di quel carteggio, ci suggerì il titolo di Rispondenze. 


\section{Riferimenti bibliografici}

Bo, Carlo (1991): «Presentazione», in Giovanna Ioli (a c. di), 18 poeti per Montale, 19811991, poesie inedite, edizione numerata, Milano, Librex.

Bruck, Edith (2017): «L'uomo Nelo Risi», in Giovanna Ioli (a c. di), Parole e immagini per Nelo Risi. Atti del convegno San Salvatore Monferrato-Alessandria, 13-14 ottobre 2016, Novara, Interlinea, pp. 29-31.

Butcher, John (2003): «Il poeta del disincanto: intervista a Nelo Risi», Resine, 95, pp. 41-46. d'Orsi, Angelo (2009): Il futurismo tra cultura e politica, reazione o rivoluzione?, Roma, Salerno Editrice.

d'Orsi, Angelo (2017): «L'ultimo libro, forse», in Giovanna Ioli (a c. di), Parole e immagini per Nelo Risi. Atti del convegno San Salvatore Monferrato-Alessandria, 13-14 ottobre 2016, Novara, Interlinea, pp. 77-82.

Dossi, Carlo (1964): Note Azzurre, a cura di Dante Isella, Milano, Adelphi.

Ferro, Pier Luigi (2008): «Un corpo collerico, isterico, disordinato per la storia del Verso libero di Gian Piero Lucini», in Gian Piero Lucini, Il verso libero, Novara, Interlinea, pp. vii-xxxvii.

Ioli, Giovanna (1995): «"Microfilm” di Andrea Zanzotto: prospettive e sguardi», Sigma [Lo sguardo, la prospettiva], XXI (4), luglio-dicembre, pp. 191-205.

Ioli, Giovanna (2008): «Rispondenze», introduzione a Nelo Risi, Né il giorno né l'ora, Milano, Mondadori.

Ioli, Giovanna (2011): «In principio furono poeti fughe e lusinghe», in Pietro Frassica (ed.), Shades of Futurism. Atti del convegno internazionale di Studi, Princeton University, 9-10 ottobre 2009, Novara, Interlinea, pp. 303-319,

Ioli, Giovanna (a c. di) (2017): Parole e immagini per Nelo Risi. Atti del convegno San Salvatore Monferrato-Alessandria, 13-14 ottobre 2016, Novara, Interlinea.

Jouve, Pierre Jean (2001): Poesie, a c. di Nelo Risi, Milano, Mondadori.

Lucini, Gian Pietro (1911 [1973]): L'ora topica di Carlo Dossi. Saggio di critica integrale, Varese, Nicola \& G [ristampa a c. di T. Grandi, Milano, Ceschina].

Lucini, Gian Pietro (1971): «Autobiografia», in Prose e canzoni amare, a c. e con introduzione di Isabella Ghidetti, Prefazione di Giorgio Luti, Firenze, Vallecchi.

Lucini, Gian Pietro (2008): Il verso libero, proposta anastatica dell'edizione 1908 di Ragion poetica e programma del verso libero: grammatica, ricordi e confidenze per servire alla storia delle lettere contemporanee, a c. di Pier Luigi Ferro, Novara, Interlinea.

Mengaldo, Pier Vincenzo (1972): Poeti italiani del Novecento, Milano, Mondadori.

Montale, Eugenio (1966 [1976]): «L'enorme anonimato in cui tutti viviamo», Corriere della sera, 6 febbraio, p. 11 [ora in E. Montale, Sulla poesia, Milano, Mondadori, pp. 150-151].

Montale, Eugenio (1996): Il secondo mestiere. Prose 1920-1979, a cura di Giorgio Zampa, t. II, Mondadori, Milano.

Risi, Nelo (1941): Le opere e i giorni (prose poetiche), Milano, Scheiwiller.

Risi, Nelo (1948): L'esperienza, Milano, Edizioni della Meridiana.

Risi, Nelo (1956): Polso teso, Milano, Mondadori.

Risi, Nelo (1958): Civilissimo, Milano, Scheiwiller.

Risi, Nelo (1961): Pensieri elementari, Milano, Mondadori.

Risi, Nelo (1965): Dentro la sostanza, Milano, Mondadori.

Risi, Nelo (1970): Di certe cose che dette in versi suonano meglio che in prosa, Milano, Mondadori. 
Risi, Nelo (1981): «Comunicato stampa Ritz cinema d'essai 1966», in G. Fofi / F. Faldini (a c. di), L'avventurosa storia del cinema italiano, Milano, Feltrinelli.

Risi, Nelo (1988): «I Contemporanei vedono se stessi», in L'Almanacco della Cometa.

Risi, Nelo (1991): Mutazioni, Milano, Mondadori.

Risi, Nelo (a c. di) (1999): Il medico di Lucini. Lettere di Arnaldo Risi, con note di Glauco Viazzi, Milano, All'Insegna del pesce d'oro di Vanni Scheiwiller.

Risi, Nelo (2000): Altro da dire, Milano, Mondadori.

Risi Nelo (2005): «Inediti», in Milo De Angelis / Antonio Riccardi (a c. di), Almanacco dello Specchio, Milano, Mondadori.

Risi, Nelo (2009): «Mondo logico e l'immaginario», in Giovanna Ioli (a c. di), Cavalcare la luce. Atti del convegno internazionale, Alessandria-San Salvatore Monferrato, 23-25 maggio 2007, Novara, Interlinea, pp 137-139.

Sanguineti, Edoardo (1998): «Lucini critico, l'oscuro e prezioso», Alias, I, 25, p. 20. 